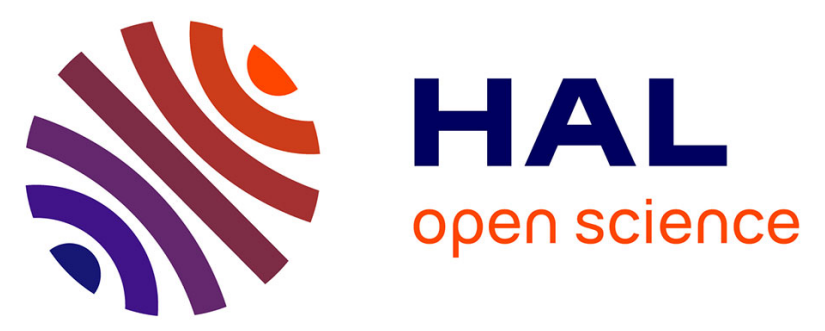

\title{
Preliminary Specificity Study of the Bestel-Clément-Sorine Electromechanical Model of the Heart using Parameter Calibration from Medical Images
}

Stéphanie Marchesseau, Hervé Delingette, Maxime Sermesant, Michel Sorine, Kawal Rhode, Simon G. Duckett, Christopher Aldo Rinaldi, Reza Razavi, Nicholas Ayache

\section{To cite this version:}

Stéphanie Marchesseau, Hervé Delingette, Maxime Sermesant, Michel Sorine, Kawal Rhode, et al.. Preliminary Specificity Study of the Bestel-Clément-Sorine Electromechanical Model of the Heart using Parameter Calibration from Medical Images. Journal of the mechanical behavior of biomedical materials, 2013, 20, pp.259-271. 10.1016/j.jmbbm.2012.11.021 . hal-00813849

\author{
HAL Id: hal-00813849 \\ https://hal.inria.fr/hal-00813849
}

Submitted on 3 Dec 2013

HAL is a multi-disciplinary open access archive for the deposit and dissemination of scientific research documents, whether they are published or not. The documents may come from teaching and research institutions in France or abroad, or from public or private research centers.
L'archive ouverte pluridisciplinaire HAL, est destinée au dépôt et à la diffusion de documents scientifiques de niveau recherche, publiés ou non, émanant des établissements d'enseignement et de recherche français ou étrangers, des laboratoires publics ou privés. 


\title{
Preliminary Specificity Study of the Bestel-Clément-Sorine Electromechanical Model of the Heart using Parameter Calibration from Medical Images
}

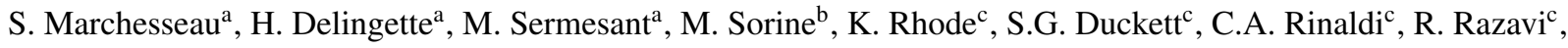 \\ N. Ayache ${ }^{a}$ \\ ${ }^{a}$ INRIA, Asclepios Research Project, Sophia Antipolis, France \\ ${ }^{b}$ INRIA, Sisyphe Research Project, Rocquencourt, France \\ ${ }^{c}$ King's College London, Division of Imaging Sciences $\mathcal{E}$ Biomedical Engineering, St. Thomas' Hospital, London, UK
}

\begin{abstract}
Patient-specific cardiac modelling can help in understanding pathophysiology and predict therapy effects. This requires the personalization of the geometry, kinematics, electrophysiology and mechanics. We use the Bestel-ClémentSorine (BCS) electromechanical model of the heart, which provides reasonable accuracy with a reduced parameter number compared to the available clinical data at the organ level. We propose a preliminary specificity study to determine the relevant global parameters able to differentiate the pathological cases from the healthy controls. To this end, a calibration algorithm on global measurements is developed. This calibration method was tested successfully on 6 volunteers and 2 heart failure cases and enabled to tune up to 7 out of the 14 necessary parameters of the BCS model, from the volume and pressure curves. This specificity study confirmed domain-knowledge that the relaxation rate is impaired in post-myocardial infarction heart failure and the myocardial stiffness is increased in dilated cardiomyopathy heart failures.
\end{abstract}

Keywords: Computer Model - Cardiac Mechanics - Specificity Analysis - Parameter Calibration

\section{Introduction}

The clinical understanding and treatment of cardiovascular diseases is highly complex. For each patient, cardiologists face issues in classifying the pathology, choosing the therapy or selecting suitable patients. In order to provide additional guidance to cardiologists, many research groups are investigating the possibility to plan such therapies based on biophysical models of the heart (Kerckhoffs, 2010). The hypothesis is that one may combine anatomical and functional data to build patient-specific cardiac models that could have the potential to predict the benefits of different therapies. Cardiac electromechanical simulations are based on computational models that can represent the heart geometry, motion and electrophysiology patterns during a cardiac cycle with sufficient accuracy. Integration of anatomical, mechanical and electrophysiological information for a given subject is essential to build such models.

Several approaches for the past 20 years have been developed to describe and simulate the cardiac func-

URL: Stephanie.Marchesseau@inria.fr (S. Marchesseau) tion, including cardiac mechanics and electrophysiology (Humphrey et al., 1990; Hunter et al., 1997; Nash, 1998; Bestel et al., 2001; Sachse, 2004). They differ in their choice of hyperelastic material, electrophysiological properties or electromechanical coupling. In this paper the Bestel-Clément-Sorine (BCS) model (Bestel et al., 2001), further improved by (Chapelle et al., 2012) is used. It showed good accuracy and predictive power under different pacing conditions in terms of haemodynamics (Sermesant et al., 2012), without being overparametrized for the available data.

The simulation becomes patient-specific after several levels of personalization: geometrical (a computational mesh is built from patient-specific anatomical images (SSFP sequence of MRI)), kinematic (the motion of the cardiac structure is estimated from cine-MR images (McLeod et al., 2012; Sermesant et al., 2006)), electrophysiological (the depolarization and repolarization times are extracted from electrocardiograms (Relan et al., 2011)) and mechanical (the mechanical parameters of the model are locally tuned so that the simulation behaves in accordance to patient-specific datasets).

The latter inverse problem has been tackled by differ- 
ent authors. For instance, (Xi et al., 2011) and (Liu and Shi, 2009) estimate the passive material stiffness with data assimilation methods while (Wang et al., 2009) use Sequential Quadratic Programming. (Moireau and Chapelle, 2011) as well as (Chabiniok et al., 2011) estimate the contractility parameters using Reduced Unscented Kalman Filtering. (Sundar et al., 2009) and (Delingette et al., 2011) rather use adjoint data assimilation methods.

These methods are efficient to estimate some model parameters from the local motion and therefore open the possibility to help diagnosis and therapy planning. However, such algorithms are dependent on the initial range of parameter values since it is often necessary to be close to the solution for the algorithm to converge towards a meaningful local solution. The choice of the parameters to estimate and their initial calibration have therefore a great impact for the personalization. Moreover, these methods give a distribution of the parameters along the myocardium which is almost impossible to analyze in order to classify or understand the pathologies. A global estimation of the parameters based on the global indices used in the clinical domain can therefore give additional guidance to the cardiologists.

The authors develop here a solution to both issues. First, since personalizing an electromechanical model requires a close initialization of the main parameters, our contribution optimizes the widely used manual calibration that precedes personalization algorithms (see Fig.1 for a description of the usual personalization pipeline). This calibration, tested on 6 volunteers cases and two heart failure cases, managed to tune 7 out of the 14 BCS model parameters with $12 \%$ relative errors. Then, the developed calibration algorithm based on the Unscented Transform (Julier and Uhlmann, 1997), assesses some space-invariant parameters from the volume and pressure evolution, leaving other parameters fixed to a standard value. The calibrated global parameters are then compared and lead to a preliminary specificity study to determine which of the 7 parameters are specific for the classification of the two heart failure cases.

The next section develops the Bestel-Clément-Sorine model (Chapelle et al., 2012) and its physiological meaning before describing the calibration method. The results section first includes some simulation results which prove that the model behaves in adequacy with the literature. Finally, the specificity study is performed using calibration results on the volunteer and control cases briefly described in (Marchesseau et al., 2012b).

\section{Materials and Methods}

\subsection{Imaging and Interventional Data Acquisition}

We demonstrate the application of the proposed method on cardiac MRI data, including both SSFP sequence for anatomical description and cine-MRI for motion tracking. Both volunteer and patient data were acquired at the Division of Imaging Sciences \& Biomedical Engineering at King's College London, UK, as part of studies that were ethically approved.

\subsubsection{Volunteer Study}

This study includes extensive multi-modality imaging of volunteers from which six healthy cases were used. All datasets consist of sequences of 4D cine-MRI with a spatial resolution of approximately $1.5 \times 1.5 \times 7 \mathrm{~mm}^{3}$ and a temporal resolution of around 30 $\mathrm{ms}$ (30 images per cardiac cycle), that cover the ventricles entirely. Volunteers were aged $28 \pm 5$ years, without clinical history of cardiac diseases. This dataset was made available to the research community for the STACOM'2011 challenge, see (Tobon-Gomez et al., 2011) for details regarding the data acquisition of this study.

\subsubsection{Patient Study}

We also used here two pathological cases from a combined imaging and endocardial mapping study concerning patients selected for CRT (Ginks et al., 2011). They were both characterized by a large QRS, due to a Left Bundle Branch Block (LBBB), and a small ejection fraction, due to the asynchronous contraction of the left ventricle and classified in NYHA class III (NYHA classes stand for the stages of heart failure according to the New York Heart Association). The first patient had a left ventricle ejection fraction of $25 \%$ and suffered from significant mechanical dysynchrony, with a QRS duration of $154 \mathrm{~ms}$ (normal QRS is less than 120ms). This patient is referred to as post myocardial infarction heart failure (post-MI HF). The second patient had a left ventricle ejection fraction of $18 \%$ and a QRS duration of $200 \mathrm{~ms}$. This patient suffered furthermore from severe dilated cardiomyopathy heart failure (DCM HF).

High quality cardiac anatomical and functional data were acquired with MRI, such as myocardial shape, wall motion and infarct sites, with a spatial resolution of approximately $1.5 \times 1.5 \times 7 \mathrm{~mm}^{3}$ and a temporal resolution of around $30 \mathrm{~ms}$. Electro-anatomical data was also obtained from catheter-based measurements that are guided using X-ray fluoroscopy with a spatial resolution of less than a centimetre and a temporal resolution close to a millisecond. Acute haemodynamic 


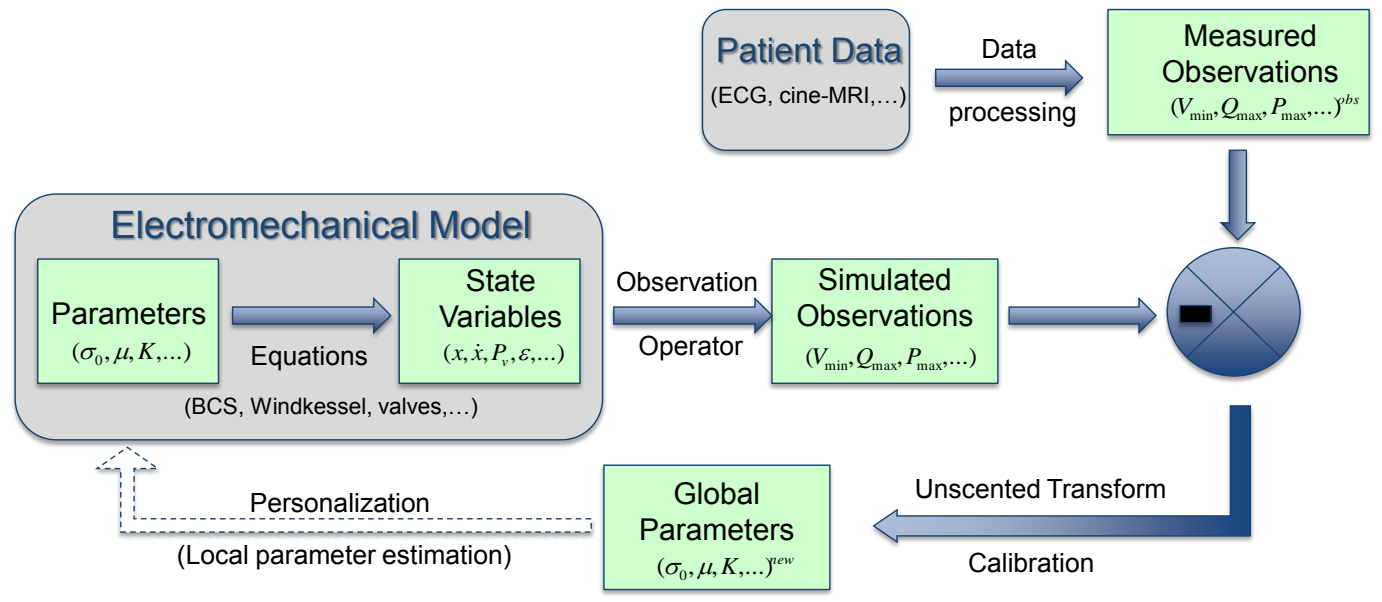

Figure 1: Complete electromechanical personalization pipeline. Our contribution does not deal with the local personalization step.

data was acquired using a high fidelity $(200 \mathrm{~Hz})$ pressure sensor to measure left ventricular pressure. This data was registered in space using the developed XMR registration (Rhode et al., 2005).

\subsubsection{Clinical Data Pre-Processing}

Three different steps are needed before any mechanical personalization can be performed: extraction of the myocardium geometry, estimation of the patient's cardiac motion and personalization of the electrophysiological propagation.

\section{Geometry Personalization}

To personalize the geometry from images, two methods were used. For the pathological cases, we combined a semi-interactive segmentation of the myocardium from the cine-MRI data at end-diastolic phase to create the 3D geometry using CardioViz3D ${ }^{1}$, and a manual correction from the SSFP sequence to include the four valves. Alternatively, we used Philips automatic cardiac segmentation (Ecabert et al., 2011) tool in GIMIAS ${ }^{2}$ to extract the ventricles from the SSFP sequence and then recreate a binary mask of the myocardium, for the healthy cases. We then used $\mathrm{CGAL}^{3}$ to create tetrahedral meshes. In the electromechanical model presented in

\footnotetext{
${ }^{1}$ CardioViz3D is an open source software for the processing, simulation and visualization of cardiac data. It is available at http://wwwsop.inria.fr/asclepios/software/CardioViz3D/

${ }^{2}$ GIMIAS is a workflow-oriented environment focused on biomedical image computing and simulation (Larrabide et al., 2009)

${ }^{3} \mathrm{C}_{\mathrm{GAL}}$ Computational Geometry Algorithms Library available at http://www.cgal.org
}

Section 2.2, fibre directions play an important role for both electrophysiological and mechanical simulations. There are several ways to generate realistic fibre directions: by mapping an atlas onto the myocardium geometry (Peyrat et al., 2007; Lombaert et al., 2011; Toussaint et al., 2010) or by synthetically varying the elevation angle (with respect to the short axis plane) across the myocardium wall. In this paper, the fibres were created synthetically with angles varying from $-70^{\circ}$ on the epicardium to $0^{\circ}$ at mid-wall to $+70^{\circ}$ on the endocardium. A thorough sensitivity analysis showed that changing extrema of elevation angles influences the global motion of the myocardium but much less the volume and pressure curves which are the observations used in the calibration process.

\section{Kinematics personalization}

A non-rigid registration algorithm was applied to the clinical 4D image sequences to find the deformation field between the end diastolic image and each subsequent image. We used the incompressible Log-Domain Demons (iLogDemons) developed by (Mansi et al., 2011) which estimates a dense non linear transformation that best aligns a template image to a reference image. Moreover, it allows to recover some components of the twist motion of the myocardium by incorporating an elastic regularizer and incompressible constraint into the registration.

\section{Electrophysiology personalization}

To simulate the electrophysiological pattern of activity, an Eikonal model was solved for the depolarization 
time $T_{d}$ at each point of the mesh: $v \sqrt{\nabla T_{d}^{t} \mathbf{D} \nabla T_{d}}=1$. $v$ is the local electrical conduction velocity and $\mathbf{D}=$ $(1-r) \mathbf{f} \otimes \mathbf{f}+r \mathbf{I}$ is the anisotropic conductivity tensor which depends on the fibre orientation $\mathbf{f}$ and on an anisotropic ratio $r$. The solution of this electrophysiological model was performed using Multi-Front Fast Marching Method (Sermesant et al., 2007). The scars tissue of post-MI HF was mapped onto the geometry from Late Enhancement images and taken into account during the electrophysiology personalization.

For healthy cases, no subject specific electrophysiological data were acquired and therefore standard values were assumed (conduction velocity of $900 \mathrm{~mm} / \mathrm{s}$ and anisotropic ratio of 0.1 ), the personalization of this model requires to specify the onset of the electrical propagation (corresponding to the extremities of the Purkinje network, on both endocardiums close to the apex). The Action Potential Duration required for the mechanical simulation was calibrated from the volume curves at the same time as the mechanical parameters for the healthy cases. For the two LBBB cases, intracardiac non contact mappings allowed to estimate the onset of depolarization as well as electrical conductivities (see (Relan et al., 2011) for further details).

\subsection{The Bestel-Clément-Sorine Electromechanical Model of the Heart}

Our approach is based on the Bestel-Clément-Sorine (BCS) model (Bestel et al., 2001) further improved by (Chapelle et al., 2012). This choice is governed by some good properties of the BCS model: It is based on a multi-scale analysis, and is compatible with the laws of thermodynamics (balance of energy may be written), which is a useful property that leads to well-posed equations of motion (Krejci et al., 2006). We describe in this section, the modelling used to represent the mechanical behaviour of the heart.

\subsubsection{Physiological Description}

The myocardium is a multi-scale structure mainly composed of myocytes (muscular cell) and extracellular matrix. A myocyte is a cell which encloses subcellular structures, sarcoplasm and myofibrils. A myofibril is divided into compartments called sarcomeres separated by Z-discs. A sarcomere is composed of actin-myosin filaments in which the binding and unbinding process occurs to lead to the contraction.

At the organ level, the ventricles are filled with blood coming from the atria and ejected through the arteries, with valves ensuring the proper circulation. The closing and opening of these valves lead to four cardiac phases (filling, isovolumetric contraction, ejection and isovolumetric relaxation).

\subsubsection{Hypotheses of the Model}

In this section we list the different modelling hypotheses (in italic font) and the mathematical formulation used to represent them, using the finite strain theory.

- The contraction of the sarcomere can be described, at the molecular scale, by the Huxley filament model (Huxley, 1957) and takes into account the Starling effect.

Statistical mechanics allows to describe the contraction at the macroscopic scale, resulting in a differential equation modeling the control of the active stress $\tau_{c}$ and stiffness $k_{c}$ by an electrical input $u$ (Chapelle et al., 2012):

$$
\left\{\begin{array}{l}
\dot{k_{c}}=-\left(|u|+\alpha\left|\dot{e_{c}}\right|\right) k_{c}+n_{0} k_{0}|u|_{+} \\
\dot{\tau_{c}}=-\left(|u|+\alpha\left|\dot{e_{c}}\right|\right) \tau_{c}+\dot{e_{c}} k_{c}+n_{0} \sigma_{0}|u|_{+}
\end{array}\right.
$$

where $\alpha$ is a constant related to the cross-bridge unbinding due to the deformation rate, $k_{0}$ and $\sigma_{0}$ are respectively the maximum stiffness and contraction. $n_{0}$ is a reduction factor that allows to take into account the Starling effect by which the maximum contraction depends on the fibre strain $e_{c}$. The control variable $u$ is derived from the electrical activation model and is a function of the free calcium concentration only (Chapelle et al., 2012). It is simplified here under the form of a function of time, by neglecting the variations in calcium dynamics (see Fig. 2):

$$
\begin{cases}u(t)=k_{A T P} & \text { for } t \in\left[T_{d}, T_{d}+A P D\right] \\ u(t)=-k_{R S} & \text { for } t \in\left[T_{d}+A P D, T_{r}\right] \\ u(t)=0 & \text { for } t \in\left[T_{r}, T_{d}+H P\right]\end{cases}
$$

$k_{A T P}$ is the rate of the myosin ATPase activity controlling the contraction rate and $k_{R S}$ is the rate of sarcoplasmic reticulum calcium re-uptake controlling the relaxation rate. $T_{d}$ and $A P D$ are respectively the depolarization time and the action potential duration (time during which the cell stays excited). $T_{d}$ is obtained by a biophysical model of cardiac electrophysiology while the APD is calibrated from the volume curves. $T_{r}$ is the time at which the active relaxation ends and the passive relaxation starts.

- The sliding of filaments creates friction in the sarcomere.

The Cauchy stress tensor for the contraction $\sigma_{c}$ is in 


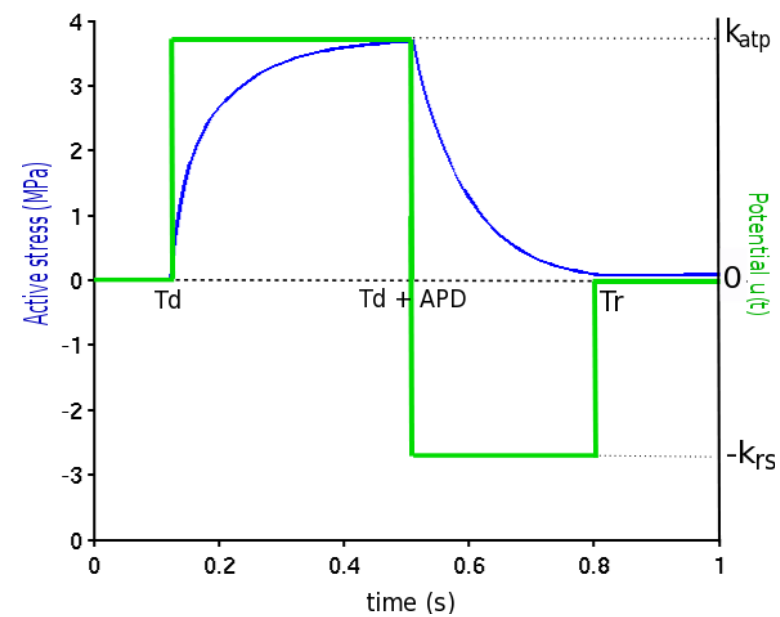

Figure 2: Active stress and potential over time

parallel with a viscosity element (cf Fig. 3) which gives

$$
\sigma_{c}=\tau_{c}+\mu \dot{e_{c}}
$$

- The Z-discs present an elastic behaviour, which enables isometric contractions.

A linear elastic component having stress $\sigma_{s}=E_{s} e_{s}$ is in series with the contractile component. Therefore, after linearization of the equations presented by (Chapelle et al., 2012), we obtain the small strain formulations $e_{1 D}=e_{s}+e_{c}$ and $\sigma_{c}=\sigma_{s}$, where $e_{1 D}$ is the projection of the Green-Lagrange deformation tensor $\mathbf{E}$ on the fibre direction: $e_{1 D}=\mathbf{f}^{T} \mathbf{E f}$.

- The extracellular matrix presents a passive hyperelastic behaviour.

Several authors (Holzapfel and Ogden, 2009; Wong et al., 2008; Mazhari and McCulloch, 2000) consider the myocardium as a whole to have an orthotropic behaviour (such as the Costa's law (Costa et al., 2001)), taking into account both fibre and laminar sheets directions. In this paper, we consider an isotropic behaviour described as a Mooney Rivlin material. Thus globally, adding the elasticity of the Z-discs, the passive behaviour of myocardial tissue in the BCS model is considered to be transversally isotropic.

An exponential hyperelastic material has been considered (Chabiniok, 2011) instead of Mooney-Rivlin but with limited benefits due to its contraction behavior. Orthotropic materials may be introduced in the future by integrating recent work from Lombaert et al. (2011) which estimates the laminar sheets direction on human hearts.
The strain energy for Mooney Rivlin material is given as:

$$
W_{e}=c_{1}\left(\bar{I}_{1}-3\right)+c_{2}\left(\bar{I}_{2}-3\right)+\frac{K}{2}(J-1)^{2}
$$

where $c_{1}, c_{2}$ are material parameters and $K$ is the Bulk modulus. The quantities $\bar{I}_{1}$ and $\bar{I}_{2}$ are the isochoric invariants of the Cauchy-deformation tensor $\mathbf{C}=\nabla \phi^{T} \nabla \phi, \bar{I}_{1}=J^{-2 / 3} I_{1}, \bar{I}_{2}=J^{-4 / 3} I_{2}$ where $I_{1}=\operatorname{tr} \mathbf{C}$, $I_{2}=\frac{1}{2}\left((\operatorname{tr} \mathbf{C})^{2}-\operatorname{tr} \mathbf{C}^{2}\right)$ and $J$ is the Jacobian $J=\operatorname{det} \nabla \phi$.

- The blood characteristics (flow, pressure) can be represented globally for each structure (atria, ventricles, arteries).

The basic circulation model is represented in Fig. 3 and defines the four phases of the cardiac cycle, independently for each ventricle as follows:

- Filling: when the left (right) ventricular pressure $P_{v}$ is smaller than the left (right) atrial pressure $P_{a t}$, the mitral (tricuspid) valve is open and the left (right) ventricle fills up with blood.

- Isovolumetric Contraction: after the contraction started, when the ventricular pressure reaches the atrial pressure, the mitral (tricuspid) valve close, therefore all valves are closed.

- Ejection: when the left (right) ventricular pressure reaches the arterial pressure, $P_{v}>P_{a r}$, the aortic (pulmonary) valve opens and the blood is ejected from the ventricle.

- Isovolumetric Relaxation: after the relaxation started, when the left (right) ventricular pressure drops below the arterial pressure, the aortic (pulmonary) valve closes and therefore all valves are closed.

To model those phases we apply the valve model introduced by (Sainte-Marie et al., 2006). It gives a relation between the blood flow leaving the ventricle $(q)$ and the atrial, ventricular and aortic pressures:

$q= \begin{cases}K_{a t}\left(P_{v}-P_{a t}\right) & \text { for } P_{v} \leq P_{a t} \\ K_{i s o}\left(P_{v}-P_{a t}\right) & \text { for } P_{a t}<P_{v} \leq P_{a r} \\ K_{a r}\left(P_{v}-P_{a r}\right)+K_{i s o}\left(P_{a r}-P_{a t}\right) & \text { for } P_{v}>P_{a r}\end{cases}$

where $K_{a t}$ and $K_{a r}$ correspond to linear laws and $K_{i s o}$ relaxes the usual isovolumetric constraint $(q=0)$. With this definition, $K_{i s o}$ is much smaller than $K_{a t}$ and $K_{a r}$. The aortic pressure is computed following the fourelement Windkessel model described in (Stergiopulos et al., 1999). This Windkessel model depends on four 

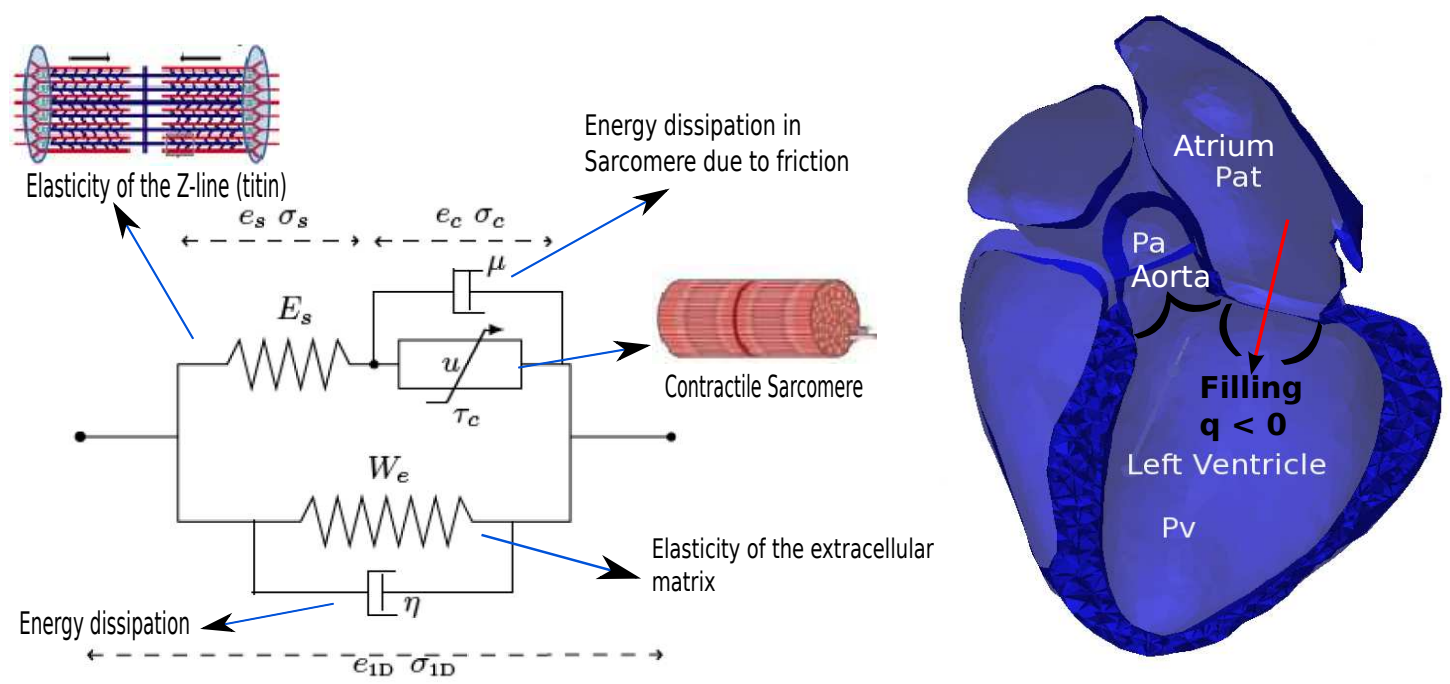

Figure 3: Full electromechanical and circulation model. (Left) $W_{e}$ is the strain energy of the extracellular matrix considered here as an isotropic material, associated with a dissipative term $\eta . u$ is a control variable which is driven by changes in transmembrane potential. It controls the contraction stress $\tau_{c} . \mu$ deals with the friction in the sarcomere while $E_{s}$ is a linear spring to enforce elasticity of the Z-discs (titin). $\left(e_{c}, \sigma_{c}\right),\left(e_{s}, \sigma_{s}\right)$ and $\left(e_{1 D}, \sigma_{1 D}\right)$ are respectively the strain and stress of the linear spring, the contraction and the fibre. (Right) Circulation model in the filling phase for the left ventricle.

parameters: the peripheral resistance $R_{p}$, the characteristic time $\tau$, the characteristic resistance $Z_{c}$ and the total arteria inertance $L$. The initial and asymptotic arterial pressures also influence the model. The evolution of atrial pressure is defined analytically as two sigmoids and depends on an initial and a maximum pressure that are set from the literature (Schäffler and Schmidt, 1999).

- The heart motion is constrained by its surrounding structures, namely the aorta, the pulmonary artery and the atria, as well as the pericardium that surrounds the myocardium.

First, the heart mesh is attached at the level of the four valve annuli limiting the ventricles. To allow some valve motion, isotropic linear springs connect the valve vertices to their initial end-diastolic positions. We define a unique stiffness matrix $\mathbf{K}=k_{b}$ Id where $k_{b}$ is the isotropic stiffness and Id is the identity matrix. Therefore the force is defined for each node as $F_{i}=\mathbf{K}\left(Q_{i}-P_{i}\right)$ where $P_{i}$ is the initial position and $Q_{i}$ is the current position. A value of $50 \mathrm{~Pa}$ was chosen for the stiffness $k_{b}$ so as to allow a small displacement of the valves. This constraint has a noticeable impact on the motion of the myocardium, but not as much on the global indices of the volume and pressure. Second, we define a fixed pericardium surface surrounding the myocardium which limits the ventricle displacements: whenever an epicardium vertex collides with the pericardial surface, a force is applied to this vertex preventing its penetration. The pericardium is defined as an offset surface of the epicardium at end-diastole with a fixed distance $(\sim 2 \mathrm{~mm})$. This collision constraint enables to limit the radial motion but does not impact the global volume or pressure evolution.

\subsection{Sensitivity Analysis of Global Outputs to the Model Parameters}

In order to select the most significant parameters given the available data, a sensitivity analysis was performed in (Marchesseau et al., 2012a). We studied the influence of each active, passive and valve model parameter on the volume and the pressure in the ventricle. We varied the parameters one by one between the ranges given in Table. 1 . This represents about 160 simulations for each ventricles. From this sensitivity analysis, we can conclude than 7 parameters are more significant than the others to characterize the global volume and pressure indices: the maximum contractility $\sigma_{0}$, the contraction rate $k_{a t p}$, the relaxation rate $k_{r s}$, the active viscosity component $\mu$, the main stiffness parameter $c_{1}$, the bulk modulus $K$ and the peripheral resistance $R_{p}$. Relevant curves are presented Fig. 4 and Fig. 5. 
Table 1: Ranges of parameter values explored in the sensitivity analysis.

\begin{tabular}{llr}
\hline Notation & Parameter Name & Min - Max \\
\hline$\sigma_{0}(\mathrm{MPa})$ & Max Contraction & $4-10$ \\
$k_{0}(\mathrm{MPa})$ & Max Stiffness & $3-9$ \\
$k_{A T P}\left(\mathrm{~s}^{-1}\right)$ & Contraction Rate & $5-20$ \\
$k_{R S}\left(\mathrm{~s}^{-1}\right)$ & Relaxation Rate & $5-60$ \\
$E_{s}(\mathrm{MPa})$ & Linear Modulus & $3-15$ \\
$\alpha$ & Cross-bridges Unfasten Rate & $0-0.8$ \\
$\mu(\mathrm{MPa} . \mathrm{s})$ & Viscosity & $0.07-0.6$ \\
$c_{1}, c_{2}(\mathrm{kPa})$ & Mooney Rivlin Modulus & $7-20$ \\
$K(\mathrm{MPa})$ & Bulk Modulus & $6-25$ \\
$\tau(s)$ & Wind. Charact. Time & $0.4-2$ \\
$R_{p}\left(\mathrm{MPa} \cdot \mathrm{m}^{-3} . \mathrm{s}\right)$ & Wind. Periph. Resistance & $30-300$ \\
$Z_{c}\left(\mathrm{MPa} . \mathrm{m}^{-3} . \mathrm{s}\right)$ & Wind. Charact. Resistance & $1-10$ \\
$L\left(\mathrm{kPa} \cdot \mathrm{s}^{2} \cdot \mathrm{m}^{-3}\right)$ & Wind. Total Art. Inertance & $1-100$ \\
\hline
\end{tabular}

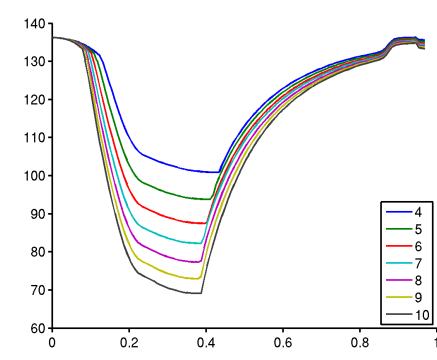

(a) $\sigma_{0}$

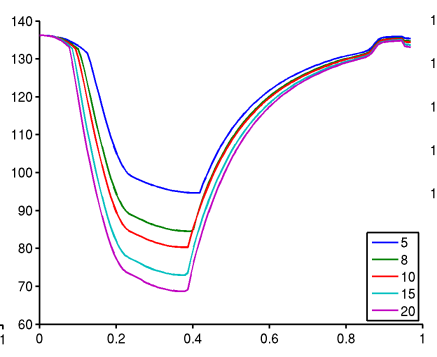

(b) $k_{A T P}$

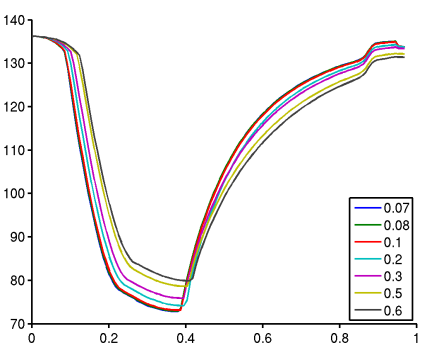

(c) $\mu$

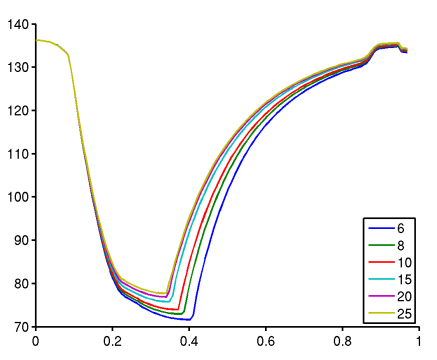

(d) $K$

Figure 4: Volume evolution (in $m L$ ) over time (in $s$ ) for the left ventricle, with varying parameters.

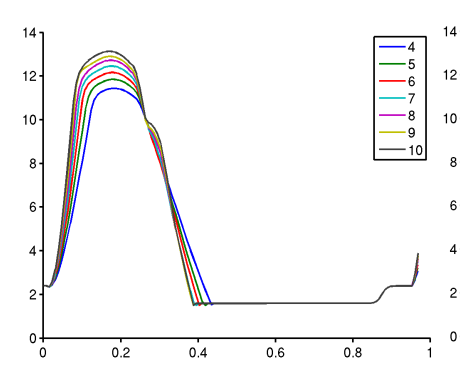

(a) $\sigma_{0}$

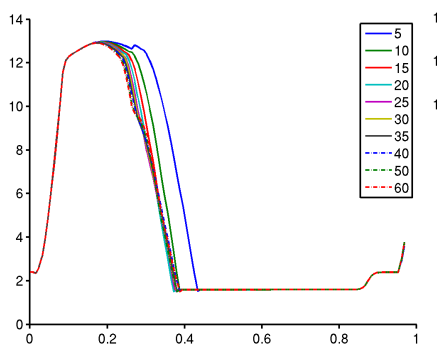

(b) $k_{R S}$

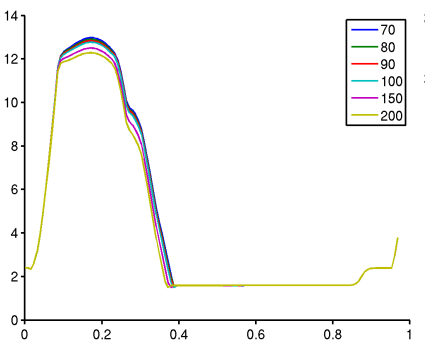

(c) $c_{1}$

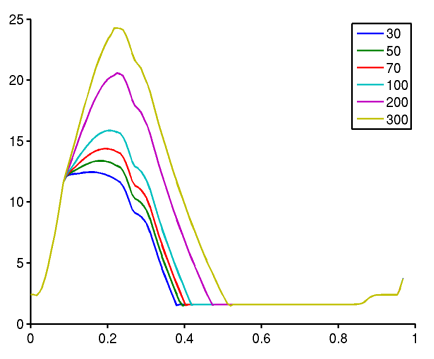

(d) $R_{p}$

Figure 5: Pressure evolution (in $k P a$ ) over time (in $s$ ) for the left ventricle, with varying parameters.

\subsection{Parameters Calibration Based on Unscented Transform}

Once selected, the sensitive parameters are calibrated from global measurements. We chose the ventricular volume and pressure curves as main observations to perform the calibration as they are important physiological indices and can be captured by few quantities: the minimum volume $V_{\min }$, the maximum and minimum of the flow ( $q_{\max }$ and $q_{\min }$ respectively) and indices on the ventricular pressure such as the maximum pressure $P_{\max }$, and the extrema of its derivative $\left(d P / d t_{\max }\right.$ and $\left.d P / d t_{\min }\right)$.

To calibrate the model, we use the algorithm derived 
from the Unscented Transform (Julier and Uhlmann, 1997). The proposed algorithm finds a set of parameters $\mathbf{X}^{\text {new }}$ that minimize the difference between the measured observation $\mathbf{Z}^{\text {obs }}$ and the predicted observation $\overline{\mathbf{Z}}$. It is explained as follows: Let $\mathbf{Z}$ be the vector of observations, here

$$
\mathbf{Z}=\left[V_{\min }, q_{\max }, q_{\min }, P_{\max }, d P / d t_{\max }, d P / d t_{\min }\right]
$$

and $\mathbf{X}$ the parameter vector which has mean $\mathbf{X}^{0}$, covariance $\mathbf{C}_{X}$ and dimension $n$. We set the covariance as $\mathbf{C}_{X}=\operatorname{Cov}(\mathbf{X}, \mathbf{X})$ by estimating the minimal and the maximal value of each parameter with a trial and error approach. We compute observations $\mathbf{Z}_{i \epsilon}$ from the $2 n+1$ sets of parameters $\mathbf{X}_{i \epsilon}=\left[x_{1}, x_{2}, \ldots, x_{i}+\epsilon s_{i}, \ldots\right]$ around the mean value $\mathbf{X}^{0}$ where $\epsilon \in\{-1,0,1\}$ and $s_{i}$ is an uncertainty function of the covariance $s_{i}=\gamma \sqrt{\mathbf{C}_{X}}$, with $\gamma$ the scaling parameters. The mean observation is set as $\overline{\mathbf{Z}}=\sum_{i, \epsilon} \omega_{i \epsilon} \mathbf{Z}_{i \epsilon}$ with some weights $\omega_{i \epsilon}$ described by (Wan and Van Der Merwe, 2000). Finally we derive the covariance matrix as:

$$
\operatorname{Cov}(\mathbf{X}, \mathbf{Z})=\sum_{i \epsilon} \omega_{i \epsilon}\left(\mathbf{X}_{i \epsilon}-\mathbf{X}^{0}\right)\left(\mathbf{Z}_{i \epsilon}-\overline{\mathbf{Z}}\right)^{T}
$$

The new set of parameters $\mathbf{X}^{\text {new }}$ found to match the observations $\mathbf{Z}^{\text {obs }}$ is

$$
\left(\mathbf{X}^{n e w}-\mathbf{X}^{0}\right)=\operatorname{Cov}(\mathbf{X}, \mathbf{Z}) \operatorname{Cov}(\mathbf{Z}, \mathbf{Z})^{-1}\left(\mathbf{Z}^{\text {obs }}-\overline{\mathbf{Z}}\right)
$$

where

$$
\operatorname{Cov}(\mathbf{Z}, \mathbf{Z})=\sum_{i \epsilon} \omega_{i \epsilon}\left(\mathbf{Z}_{i \epsilon}-\overline{\mathbf{Z}}\right)\left(\mathbf{Z}_{i \epsilon}-\overline{\mathbf{Z}}\right)^{T}
$$

This algorithm is very simple to implement and runs in one iteration to give $\mathbf{X}^{\text {new }}$. Another simulation is necessary to obtain the resulting observation $\mathbf{Z}^{\text {new }}$. The algorithm is illustrated in Fig. 6.

\subsection{Specificity Analysis}

The calibration algorithm is used to compare the estimated parameters between volunteer and heart failure cases. Both volunteer and pathological data include the MRI sequences. From the kinematic personalization, we registered all images to the end diastolic image. Then, image transformations, stored as displacement fields, were applied to the end-diastolic tetrahedral mesh to estimate the volume of the ventricles over time. Moreover, the heart failure cases data includes the pressure curves, therefore all 6 observations were available: the minimum volume $V_{\min }$, the extrema of the outward flow $Q_{\min }$ and $Q_{\max }$, the maximum pressure $P_{\max }$ and the

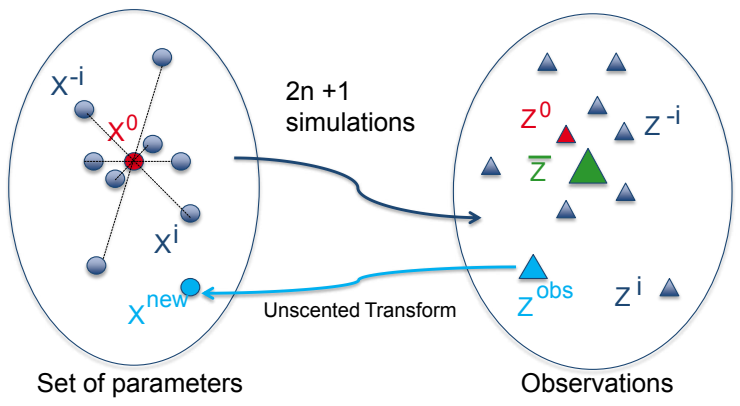

Figure 6: Schematic representation of the Unscented Transform algorithm

extrema of its derivative $d P / d t_{\text {min }}$ and $d P / d t_{\text {max }}$. Volunteer data included only MR images, so only the volume evolution is available. Therefore standard pressure indices were imposed on the volunteers so that the same calibration technique could be performed on control and pathological cases. From this comparison, conclusions can be extracted on which parameter is specific to the type of disease. Results of this study are presented in Sec. 3.3. The calibration of the $A P D$ enabled a better fit of the cardiac phases but is not considered here for the specificity analysis.

\section{Results}

\subsection{Forward Simulation of the Bestel-Clement-Sorine Model}

The simulations were performed on a laptop PC with a Intel Core Duo processor at $2.80 \mathrm{~Hz}$ and took around 10 minutes per cardiac cycle for meshes with approximately 80,000 tetrahedra. The time steps were set depending on the cardiac cycles and the number of images (for instance $d t=7.75 \mathrm{~ms}$ for 30 images and a heart period of $0.93 \mathrm{~s}$ ). A sensitivity study on the mesh quality and the time step showed that the chosen values lead to a good trade-off between computation time and accuracy. An example of resulting curves for the pressure and the volume in the left ventricle is given in Fig. 7 including the four cardiac phases. The mesh and the electrophysiological mapping used for this simulation are also presented in Fig. 7. With this example, the power developed by the heart can be computed as $P=\Delta V_{L} \Delta P_{v L} \approx 1.3 \mathrm{~W}$ which is in the range of $[1 W, 2 W]$ usually referenced in the literature. Moreover, effects of preload (increase of the atrial pressure), afterload (increase of the aortic pressure) and inotropy (increase of the contraction rate) on Pressure-Volume 


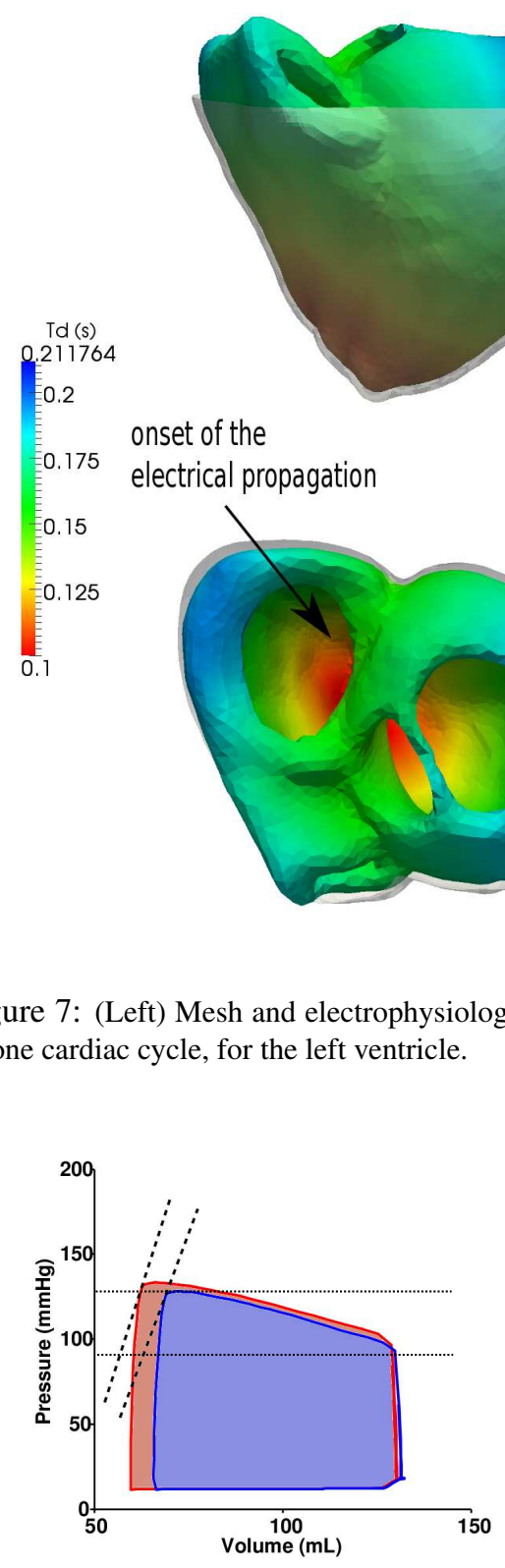

(a) Inotropy

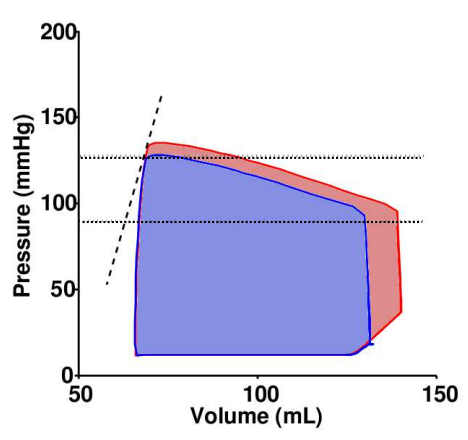

(b) Preload

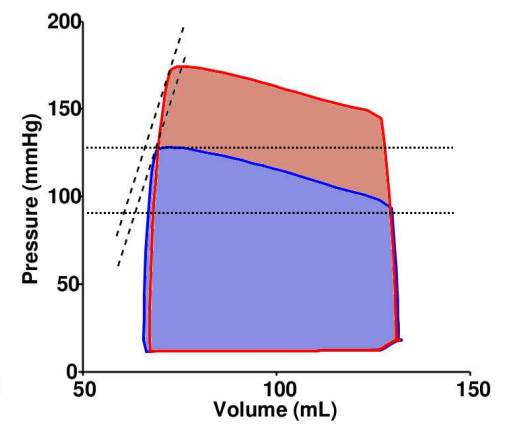

(c) Afterload

Figure 8: Independent increase of inotropy, preload and afterload (red loop), in comparison with the normal loop (blue). Horizontal dotted lines represent normal aortic systolic and diastolic pressures while dashed lines represent the slopes of the end-systolic pressure-volume relationship.

loops were studied. Fig. 8 shows the results of these tests, which are in agreement with (Klabunde, 2011).

\subsection{Calibration Results on Healthy and Pathological cases}

The proposed calibration algorithm was then applied on the volunteers and the two Left Bundle Branch
Block (LBBB) cases after performing kinematics and electrophysiology personalization, for the parameter vector $\mathbf{X}=\left[\sigma_{0}, k_{a t p}, k_{r s}, \mu, c_{1}, K, R_{p}\right]$ with initial value of the parameters $\mathbf{X}^{0}=[7,15,35,0.28,80,13,100]$. A calibration on the left ventricle only was performed due to the difficulty to segment and register the right ventricle leading to unreliable volume curves. 
The relative errors between the real observations $\mathbf{Z}^{\text {obs }}$ and the simulated observations $\mathbf{Z}^{\text {new }}$ are shown on Fig.11, before and after calibration. A comparison of the simulated mesh and the MRI sequence for the LBBB post-MI HF case is presented Fig. 12. It shows that the calibration gives satisfactory results and can therefore be used for the preliminary specificity study. Differences noticed on images b) and c) are primarily due to the registration (observation) errors, the calibration errors and also the model hypotheses (e.g. constant parameters over the myocardium). Fig. 9 shows the measured, reference and estimated volume curves on one volunteer and Fig. 10 shows the results of the LBBB post-MI HF case.

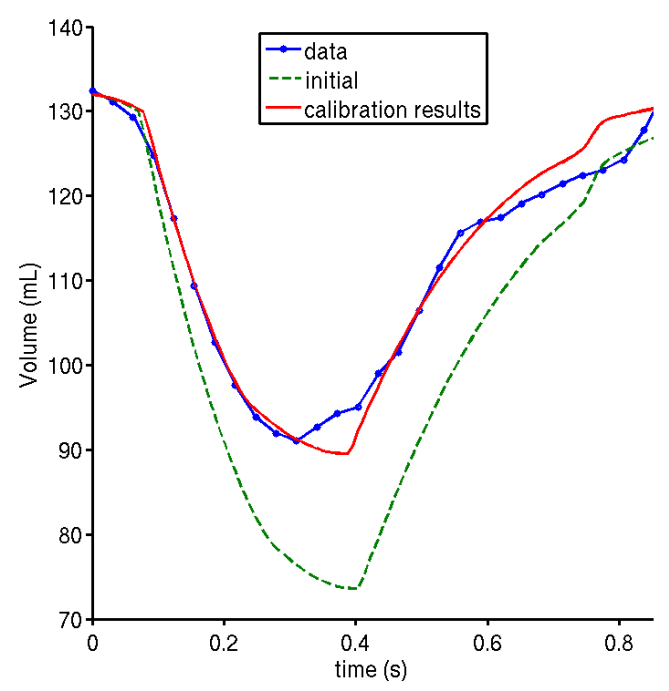

Figure 9: Results of the calibration technique on real data for one healthy volunteer.

\subsection{Preliminary Specificity Analysis}

A box plot in Fig. 13 presents the results of all volunteer cases on parameter values normalized by $\mathbf{X}^{0}$ through their five-number summaries: the smallest value, lower quartile, median (red), upper quartile, and largest value. We can easily see that for some model parameters, the values obtained for the pathological cases are outliers compared to the values of the volunteers. In more details, it shows that the post-MI HF case has a reduced peak contractility and an impaired relaxation (reduced relaxation rate). It is in agreement with medical knowledge about myocardial infarction impact on diastole, for instance such decrease of the relaxation rate was evaluated in ischemic dog hearts (Williams et al.,
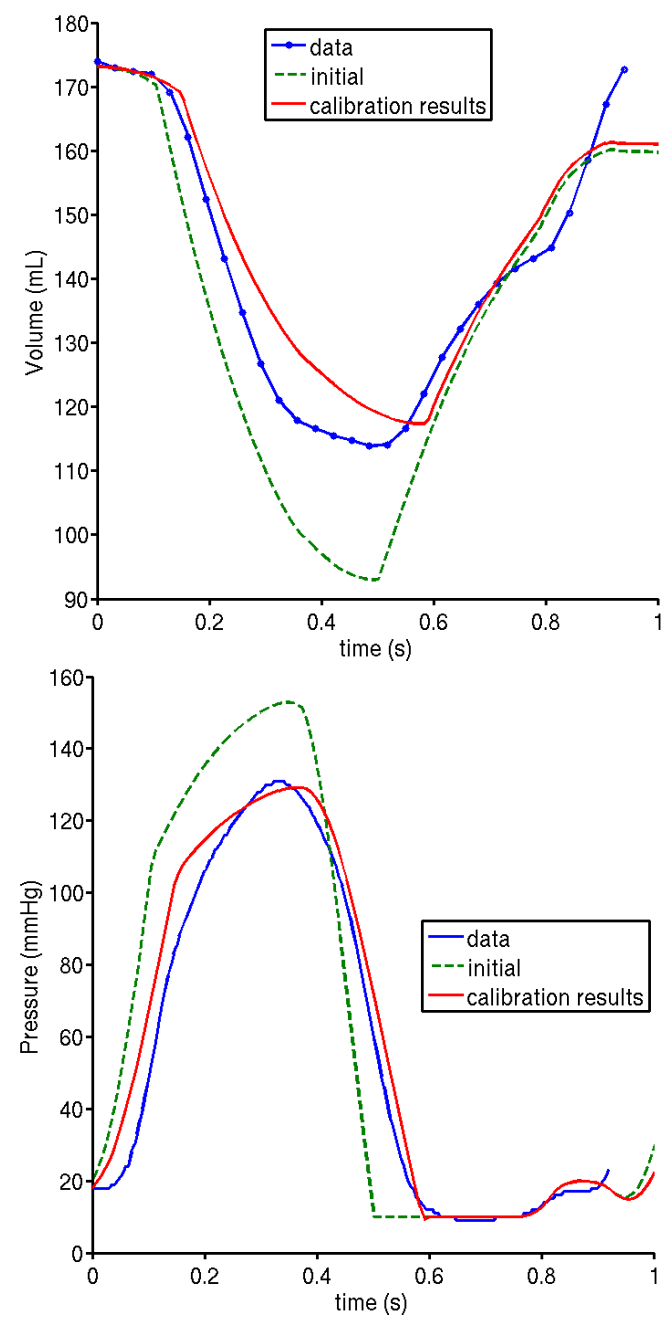

Figure 10: Results of the calibration technique on real data for post-MI HF case.

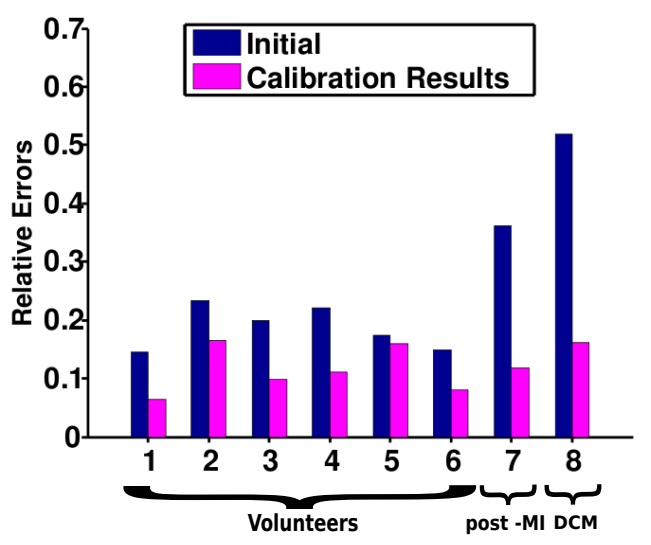

Figure 11: Relative errors on the observations of the 6 volunteers and the two LBBB cases. 


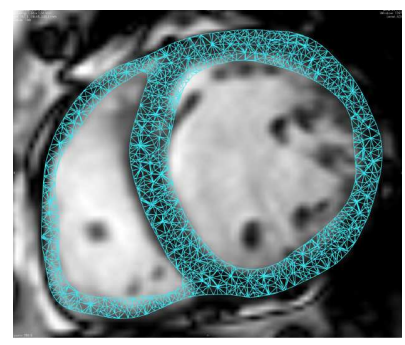

(a) $\mathrm{t}=0 \mathrm{~ms}$

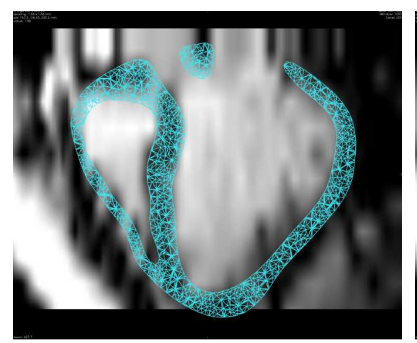

(e) $\mathrm{t}=0 \mathrm{~ms}$

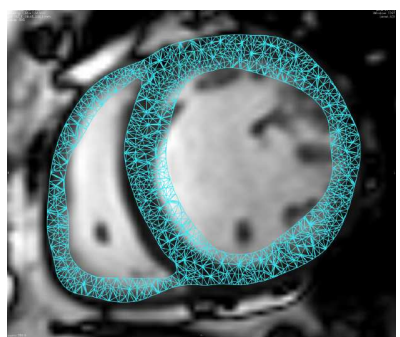

(b) $\mathrm{t}=190 \mathrm{~ms}$

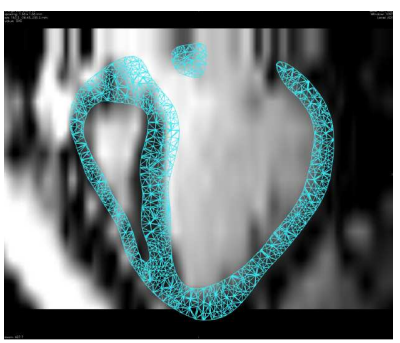

(f) $\mathrm{t}=190 \mathrm{~ms}$

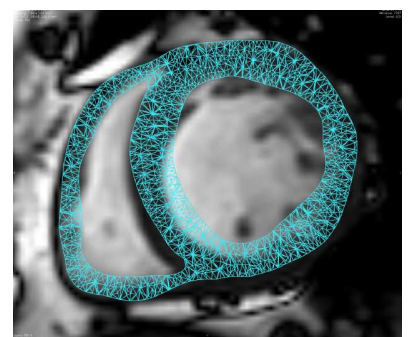

(c) $\mathrm{t}=410 \mathrm{~ms}$

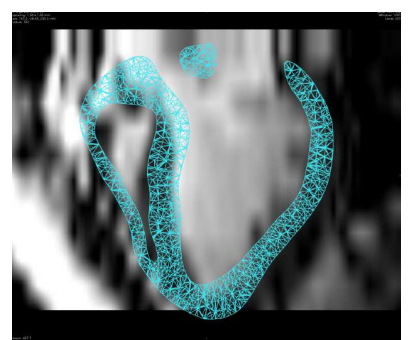

(g) $\mathrm{t}=410 \mathrm{~ms}$

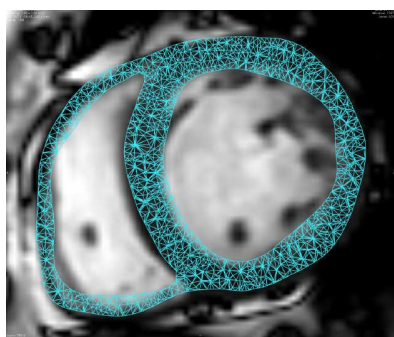

(d) $\mathrm{t}=720 \mathrm{~ms}$

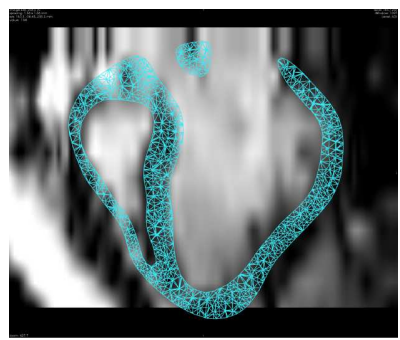

(h) t $=720 \mathrm{~ms}$

Figure 12: Short axis and long axis views of the simulated mesh on top of the MR images at different moments of the cardiac cycle (heart period $=930 \mathrm{~ms}$ ) for LBBB post-MI HF case.

1980). The dilated cardiomyopathy patient with heart failure has also a reduced peak contractility with a much higher myocardial stiffness. This is also in agreement with the literature, where such differences in myocardial stiffness have been observed in DCM patients (Bortone et al., 1989). We also observe a smaller aortic resistance in this case, which is a desired effect of the vasodilator treatment the DCM patient had undergone.

Therefore this preliminary study could assess

- the small relaxation rate $k_{r s}$ as a characteristic of post-MI heart;

- the high stiffness $c_{1}$ and bulk modulus $K$ as a possible clue of severe dilated cardiomyopathy;

- the small contractility $\sigma_{0}$ as a possible indication of heart failures.

The simulated Pressure-Volume diagrams are presented Fig. 14 and demonstrate the clear differences between volunteer cases and the heart failure cases.

\section{Discussion}

We presented the simulation of an electromechanical model of the heart with a complexity compatible with clinical data and a preliminary specificity analysis based

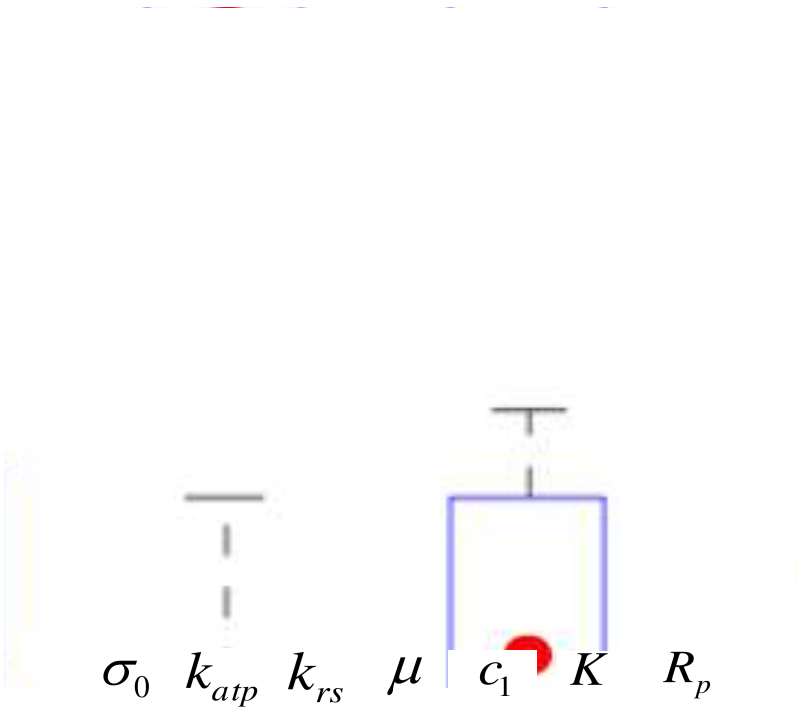

Figure 13: Box plot showing the median and variance of the parameters for seven healthy cases. The values are normalized by the mean parameter $\mathbf{X}^{0}$. The red and blue dots represent the parameters of the pathological cases. 


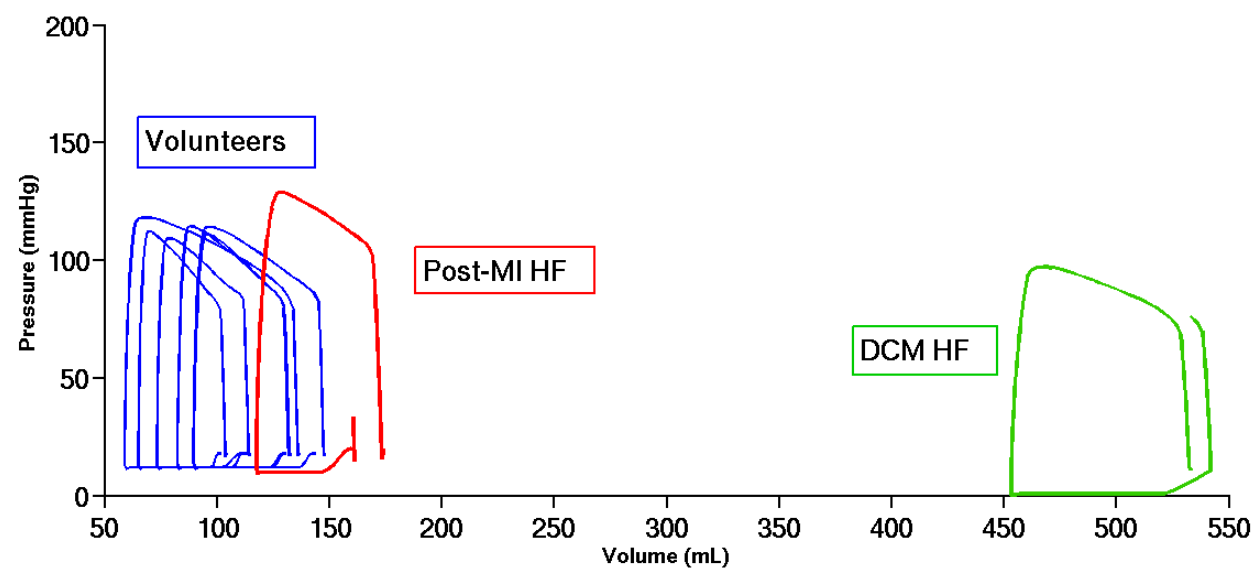

Figure 14: Simulated Pressure-Volume diagrams after calibration.

on global parameter calibration from volume and pressure indices.

\subsection{The Bestel-Clément-Sorine model}

The choice of this model to characterize the mechanical behaviour of the heart has been justified in several ways. First, the hypotheses that constitute the model are all physiologically relevant and do not input any $a$ priori knowledge on the observations (for instance the pressure). Also, the model depends on only 14 global parameters from which 7 were easily tuned from global indices of the volume and pressure curves. The ratio between the number of sensitive parameters and the total number of parameters is therefore good, which confirms the quality of this model for such application. Moreover, tests on preload, afterload and inotropy changes demonstrated that the model behaves in agreement with literature in such conditions (Klabunde, 2011). Finally, the preliminary study gave results in agreement with medical knowledge and literature proving once more the physiological behavior of this model. The authors believe that this model, one of the most complex models that have been personalized (Sermesant et al., 2012), fits the requirements to represent pathological and healthy cardiac behaviors while being compact enough for personalization.

The fiber field was simplified in this work due to its small impact on the 1D measurements. However, in future work, the 3D local motion will be studied therefore the creation of the fiber field will be improved using the now available human atlas Lombaert et al. (2011).

\subsection{From Global Calibration to Regional Personaliza- tion Methods}

We proposed in this paper a calibration algorithm based on the Unscented Transform that allowed us to tune 7 global parameters from volume and pressure curves, with a mean relative error of $11 \%$, in one iteration. Several iterations were tested and did not improve the results which shows that the model cannot fit the registered curve better than what we found. This can be due to registration or/and modelling errors. Moreover, the goal of the calibration is to automatically and quickly initialize global parameters before regional personalization, therefore a more complex algorithm would not only lead to similar results but would probably be less applicable to that many cases.

Since the calibration requires only to run several simulations in parallel to estimate these parameters followed by one additional simulated cycle to verify the results, it can easily be used as a preprocessing step before the application of more sophisticated personalization algorithms. Moreover, the method gives good results using the same initial value $\mathbf{X}^{0}$ and the same sigma points for all the cases, which decreases the preprocessing time and enables a comparison of the calibrated parameters. However, results for the pathological cases could be improved by a better choice of the initial value $\mathbf{X}^{0}$. Studying more cases would help in defining the proper initial values in the different pathological cases.

Moreover, volume and pressure curves provide basic information about the cardiac cycle. A larger set of parameters could be personalized by adding global or even regional motion indices such as radial, longitudinal or circumferential displacements or strains in the ventricles or AHA segments (defined by the American Heart 
Association). Further work will include these indices, using data assimilation methods from different imaging modalities (tagged-MRI or cine-MRI).

\subsection{Specificity Study}

A preliminary study showed that results on pathological cases versus control cases were coherent with medical knowledge of the studied diseases. We can therefore give a first set of specific parameters to characterize these heart failure cases. In our cases, a small relaxation rate was found specific to ischemic heart failures, and a high passive stiffness was specific to a severe dilated cardiomyopathy. Moreover, the results confirmed that a small contractility is expected in heart failure cases. Additional pathological cases and observation quantities are required to further develop and validate the specificity study.

\section{Conclusion}

To conclude, we proposed an efficient calibration algorithm that enabled to assess up to 7 global physiological parameters of the Bestel-Clément-Sorine model. Moreover, the performed preliminary specificity study gave results in accordance with the medical findings for dilated cardiomyopathy and ischemic heart failure cases. The authors believe that these techniques, once further validated, could help in classifying and detecting cardiac pathologies, as well as providing a first step for further local personalization.

\section{Acknowledgment}

This work is partially funded by the European project euHeart (http://www.euheart.eu) FP7-2007-2013224495 and by the ERC advanced Grant MedYMA. The authors warmly thank Kristin McLeod, Adityo Prakosa, Loïc Le Folgoc, Ján Margeta and Jatin Relan from Asclepios team for their support and constructive discussions, as well as Radomir Chabiniok, Dominique Chapelle, for their expertise on the model.

\section{References}

Bestel, J., Clement, F., Sorine, M., 2001. A biomechanical model of muscle contraction. Medical Image Computing and Computer Assisted Intervention (MICCAI), 1159-1161.

Bortone, A. S., Hess, O. M., Chiddo, A., Gaglione, A., Locuratolo, N., Caruso, G., Rizzon, P., 1989. Functional and structural abnormalities in patients with dilated cardiomyopathy. J Am Coll Cardiol 14, 613623.

Chabiniok, R., 2011. Personalized biomechanical heart modeling for clinical applications. Ph.D. thesis, Université Pierre et Marie Curie - Paris 6.
Chabiniok, R., Moireau, P., Lesault, P., Rahmouni, A., Deux, J., Chapelle, D., 2011. Estimation of tissue contractility from cardiac cine-MRI using a biomechanical heart model. Biomechanics and Modeling in Mechanobiology, 1-22.

Chapelle, D., Le Tallec, P., Moireau, P., Sorine, M., 2012. An energypreserving muscle tissue model: formulation and compatible discretizations. International Journal for Multiscale Computational Engineering 10 (2), 189-211.

Costa, K. D., Holmes, J., McCulloch, A. D., 2001. Modelling cardiac mechanical properties in three dimensions. Phil. Trans. R. Soc. Lond. 359, 1233-1250.

Delingette, H., Billet, F., Wong, K. C. L., Sermesant, M., Rhode, K., Ginks, M., Rinaldi, C. A., Razavi, R., Ayache, N., 2011. Personalization of Cardiac Motion and Contractility from Images using Variational Data Assimilation. IEEE transactions on bio-medical engineeringIn Press.

Ecabert, O., Peters, J., Walker, M. J., Ivanc, T., Lorenz, C., von Berg, J., jonathan Lessick, mani Vembar, Weese, J., December 2011. Segmentation of the heart and great vessels in CT images using a model-based adaptation framework. Medical Image Analysis $15(6), 863-876$.

Ginks, M. R., Lambiase, P. D., Duckett, S. G., Bostock, J., Chinchapatnam, P., Rhode, K., McPhail, M. J., Simon, M., Bucknall, C., Carr-White, G., Razavi, R., Rinaldi, C. A., Mar 2011. A simultaneous X-Ray/MRI and noncontact mapping study of the acute hemodynamic effect of left ventricular endocardial and epicardial cardiac resynchronization therapy in humans. Circulation Heart Failure 4 (2), 170-179.

Holzapfel, G., Ogden, R., 2009. Constitutive modelling of passive myocardium: a structurally based framework for material characterization. Philosophical Transactions of the Royal Society A: Mathematical, Physical and Engineering Sciences 367 (1902), 3445-3475.

Humphrey, J., Strumpf, R., Yin, F., 1990. Determination of a constitutive relation for passive myocardium: I. a new functional form. Journal of biomechanical engineering 112, 333.

Hunter, P., Nash, M., Sands, G., 1997. Computational electromechanics of the heart. Computational biology of the heart, 345-407.

Huxley, A., 1957. Muscle structure and theories of contraction. Progress in biophysics and biophysical chemistry 7, 255.

Julier, S., Uhlmann, J., 1997. A new extension of the Kalman filter to nonlinear systems. In: Int. Symp. Aerospace/Defense Sensing, Simul. and Controls. Vol. 3. Citeseer, p. 26.

Kerckhoffs, R. C. P. (Ed.), 2010. Patient-Specific Modeling of the Cardiovascular System, Technology-Driven Personalized Medicine. Springer.

Klabunde, R., 2011. Cardiovascular physiology concepts. Lippincott Williams \& Wilkins.

Krejci, P., Sainte-Marie, J., Sorine, M., Urquiza, J.-M., September 2006. Solutions to muscle fiber equations and their long time behaviour. Nonlinear Analysis: Real World Applications 7 (4), 535558.

Larrabide, I., Omedas, P., Martelli, Y., Planes, X., Nieber, M., Moya, J., Butakoff, C., Sebastián, R., Camara, O., De Craene, M., et al., 2009. Gimias: an open source framework for efficient development of research tools and clinical prototypes. Functional Imaging and Modeling of the Heart, 417-426.

Liu, H., Shi, P., 2009. Maximum a posteriori strategy for the simultaneous motion and material property estimation of the heart. Biomedical Engineering, IEEE Transactions on 56 (2), 378-389.

Lombaert, H., Peyrat, J.-M., Croisille, P., Rapacchi, S., Fanton, L., Clarysse, P., Delingette, H., Ayache, N., May 2011. Statistical Analysis of the Human Cardiac Fiber Architecture from DT-MRI. In: Axel, L., Metaxas, D. (Eds.), Proceedings of FIMH Conference 2011. Vol. 6666 of LNCS. Springer, pp. 171-179, best Paper 
Award.

Mansi, T., Pennec, X., Sermesant, M., Delingette, H., Ayache, N., 2011. iLogDemons: A demons-based registration algorithm for tracking incompressible elastic biological tissues. International Journal of Computer Vision 92 (1), 92-111.

Marchesseau, S., Delingette, H., Sermesant, M., Ayache, N., 2012a. Fast parameter calibration of a cardiac electromechanical model from medical images based on the unscented transform. Biomechanics and Modeling in Mechanobiology.

Marchesseau, S., Delingette, H., Sermesant, M., Rhode, K., Duckett, S., Rinaldi, C., Razavi, R., Ayache, N., October 2012b. Cardiac mechanical parameter calibration based on the unscented transform. In: Proceedings of Medical Image Computing and Computer Assisted Intervention 2012 (MICCAI). Vol. 7511 of LNCS. Springer, Heidelberg.

Mazhari, R., McCulloch, A. D., 2000. Effect of laminar orthotropic myofiber architecture on regional stress and strain in the canine left ventricule. Journal of Elasticity 61 (4), 143-164.

McLeod, K., Prakosa, A., Mansi, T., Sermesant, M., Pennec, X., September 2012. An Incompressible Log-Domain Demons Algorithm for Tracking Heart Tissue. In: Proc. MICCAI Workshop on Statistical Atlases and Computational Models of the Heart: Mapping Structure and Function (STACOM11). Vol. 7085 of LNCS. Springer, Toronto, pp. 54-65, in press.

Moireau, P., Chapelle, D., 2011. Reduced-order Unscented Kalman Filtering with application to parameter identification in large-dimensional systems. COCV 17, 380-405, doi:10.1051/cocv/2010006.

Nash, M., 1998. Mechanics and material properties of the heart using an anatomically accurate mathematical model. Ph.D. thesis, University of Auckland.

Peyrat, J.-M., Sermesant, M., Pennec, X., Delingette, H., Xu, C., McVeigh, E. R., Ayache, N., November 2007. A Computational Framework for the Statistical Analysis of Cardiac Diffusion Tensors: Application to a Small Database of Canine Hearts. IEEE Transactions on Medical Imaging 26 (11), 1500-1514, pMID: 18041265.

Relan, J., Chinchapatnam, P., Sermesant, M., Rhode, K., Ginks, M., Delingette, H., Rinaldi, C. A., Razavi, R., Ayache, N., 2011. Coupled Personalization of Cardiac Electrophysiology Models for Prediction of Ischaemic Ventricular Tachycardia. Journal of the Royal Society Interface Focus 1 (3), 396-407.

Rhode, K., Sermesant, M., Brogan, D., Hegde, S., Hipwell, J., Lambiase, P., Rosenthal, E., Bucknall, C., Qureshi, S., Gill, J., Razavi, R., Hill, D., 2005. A system for real-time XMR guided cardiovascular intervention. IEEE Transactions on Medical Imaging 24 (11) 1428-1440.

Sachse, F., 2004. Computational cardiology: modeling of anatomy, electrophysiology, and mechanics. Vol. 2966. Springer Verlag.

Sainte-Marie, J., Chapelle, D., Cimrman, R., Sorine, M., 2006. Modeling and estimation of the cardiac electromechanical activity. Computers \& structures 84 (28), 1743-1759.

Schäffler, A., Schmidt, S., 1999. Anatomie Physiologie Biologie. In French.

Sermesant, M., Chabiniok, R., Chinchapatnam, P., Mansi, T., Billet, F., Moireau, P., Peyrat, J., Wong, K., Relan, J., Rhode, K., Ginks, M., Lambiase, P., Delingette, H., Sorine, M., Rinaldi, C., Chapelle, D., Razavi, R., Ayache, N., 2012. Patient-specific electromechanical models of the heart for the prediction of pacing acute effects in CRT: A preliminary clinical validation. Medical Image Analysis $16(1), 201-215$

Sermesant, M., Delingette, H., Ayache, N., 2006. An Electromechanical Model of the Heart for Image Analysis and Simulation. IEEE Transactions in Medical Imaging 25 (5), 612-625.

Sermesant, M., Konukoglu, E., Delingette, H., Coudiere, Y., Chin- chaptanam, P., Rhode, K., Razavi, R., Ayache, N., 7-9 June 2007. An anisotropic multi-front fast marching method for real-time simulation of cardiac electrophysiology. In: Proceedings of Functional Imaging and Modeling of the Heart 2007 (FIMH'07). Vol. 4466 of LNCS. pp. 160-169.

Stergiopulos, N., Westerhof, B., Westerhof, N., 1999. Total arterial inertance as the fourth element of the windkessel model. American Journal of Physiology-Heart and Circulatory Physiology 276 (1), H81.

Sundar, H., Davatzikos, C., Biros, G., 2009. Biomechanicallyconstrained 4D estimation of myocardial motion. Medical Image Computing and Computer-Assisted Intervention-MICCAI 2009, 257-265.

Tobon-Gomez, C., De Craene, M., Dahl, A., Kapetanakis, S., CarrWhite, G., Lutz, A., Rasche, V., Etyngier, P., Kozerke, S., Schaffeter, T., Riccobene, C., Martelli, Y., Camara, O., Frangi, A., Rhode, K. S., September 2011. A multimodal database for the 1st cardiac motion analysis challenge. In: Proc. MICCAI Workshop on Statistical Atlases and Computational Models of the Heart: Mapping Structure and Function (STACOM11). Vol. 7085 of LNCS. Springer, Toronto, pp. 32-43, in press.

Toussaint, N., Stoeck, C. T., Kozerke, S., Sermesant, M., Batchelor, P. G., September 2010. In-vivo Human 3D Cardiac Fibre Architecture: Reconstruction Using Curvilinear Interpolation of Diffusion Tensor Images. In: Proc. Medical Image Computing and Computer Assisted Intervention (MICCAI'10). Vol. 13 of LNCS. Springer, Beijing, China, pp. 418-425.

Wan, E., Van Der Merwe, R., 2000. The unscented Kalman filter for nonlinear estimation. In: Adaptive Systems for Signal Processing, Communications, and Control Symposium 2000. AS-SPCC. The IEEE 2000. IEEE, pp. 153-158.

Wang, V., Lam, H., Ennis, D., Cowan, B., Young, A., Nash, M., 2009. Modelling passive diastolic mechanics with quantitative MRI of cardiac structure and function. Medical Image Analysis 13 (5), 773-784.

Williams, E., Kaplan, J., Thatcher, F., Zimmerman, G., Knoebel, S., 1980. Prolongation of proton spin lattice relaxation times in regionally ischemic tissue from dog hearts. Journal of Nuclear Medicine $21(5), 449$.

Wong, K. C., Wang, L., Zhang, H., Liu, H., Shi, P., 2008. Simulation of Active Cardiac Dynamics with Orthotropic Hyperelastic Material Model. The MIDAS Journal - Computational Biomechanics for Medicine.

Xi, J., Lamata, P., Lee, J., Moireau, P., Chapelle, D., Smith, N., 2011. Myocardial transversely isotropic material parameter estimation from in-silico measurements based on a reduced-order unscented Kalman filter. Journal of the Mechanical Behavior of Biomedical Materials. 